\title{
CONVERGENCE AND STABILITY OF THE THREE-STEP ITERATIVE SCHEMES FOR A CLASS OF GENERAL QUASIVARIATIONAL-LIKE INEQUALITIES
}

\author{
ZEQING LIU, ZHEFU AN, SHIN MIN KANG, \\ and JEONG SHEOK UME
}

Received 27 April 2004

\begin{abstract}
We introduce and study a class of general quasivariational-like inequalities in Hilbert spaces, suggest two general algorithms, and establish the existence and uniqueness of solutions for these kinds of inequalities. Under certain conditions, we discuss convergence and stability of the three-step iterative sequences generated by the algorithms.
\end{abstract}

2000 Mathematics Subject Classification: 47J20, 49J40.

1. Introduction. In the last 30 years, variational inequalities have made much developments in the theory and applications, see, for example, [1, 3, 4, 6, 7, 8, 9] and the references therein. Recently Ding and Luo [1], Hassouni and Moudafi [3], Huang [4], Liu, et al. [6], and others introduced and studied some classes of varitional inequalities and variational-like inclusions in Hilbert spaces, proved the existence and uniqueness of solutions, and developed some new perturbed iterative algorithms for finding the approximate solutions for these variational inequalities and variational-like inclusions.

In this paper, we introduce and study a class of general quasivariational-like inequalities in Hilbert spaces, suggest three-step perturbed iterative algorithms, and establish the existence and uniqueness of solutions for these kinds of inequalities. Under certain conditions, we discuss convergence and stability of the three-step perturbed iterative algorithms. Our results extend, improve, and unify the results due to Ding and Luo [1], Hassouni and Moudafi [3], and Huang [4].

Throughout this paper, we assume that $H$ is a real Hilbert space endowed with the norm $\|\cdot\|$ and inner product $\langle\cdot, \cdot\rangle$, respectively, and $\mathbb{R}=(-\infty, \infty)$. Let $A, B, C, g, m: H \rightarrow$ $H$ and $\eta: H \times H \rightarrow H$ be mappings and let $\phi: H \times H \rightarrow \mathbb{R} \cup\{+\infty\}$ be a proper functional such that for each fixed $y \in H, \phi(\cdot, y): H \rightarrow \mathbb{R} \cup\{+\infty\}$ is lower semicontinuous and $\eta$-subdifferentiable and $(g-m)(H) \cap \operatorname{dom} \partial \phi(\cdot, y) \neq \varnothing$. We now consider the following general quasivariational-like inequality problem.

Find $x \in H$ such that $(g-m)(x) \in \operatorname{dom} \partial \phi(\cdot, x)$ and

$$
\begin{aligned}
& \langle A(x)-(B(x)-C(x)), \eta(y,(g-m)(x))\rangle \\
& \quad \geq \phi((g-m)(x), x)-\phi(y, x) \quad \forall y \in H,
\end{aligned}
$$

where $(g-m)(y)=g(y)-m(y)$ for all $x \in H$. 
SPECIAL CASES. (a) If $C(x)=m(x)=0$ for all $x \in H$, then problem (1.1) reduces to finding $x \in H$ such that $g(x) \in \operatorname{dom} \partial \phi(\cdot, x)$ and

$$
\langle A(x)-B(x), \eta(y, g(x))\rangle \geq \phi(g(x), x)-\phi(y, x) \quad \forall y \in H,
$$

which is called the general quasivariational-like inclusion, introduced and studied by Ding and Luo [1].

(b) If $C(x)=m(x)=0, \eta(y, x)=y-x$, and $\phi(x, y)=\phi(x)$ for all $x, y \in H$, then problem (1.1) reduces to determining $x \in H$ such that $g(x) \in \operatorname{dom} \partial \phi(x)$ and

$$
\langle A(x)-B(x), y-g(x)\rangle \geq \phi(g(x))-\phi(y) \quad \forall y \in H .
$$

This concept was introduced and studied by Hassouni and Moudafi [3] and Huang [4].

Next we recall some definitions and notations.

DEFINITION 1.1 [1]. Let $\eta: H \times H \rightarrow H$ be a mapping. A proper functional $\phi: H \rightarrow$ $\mathbb{R} \cup\{+\infty\}$ is said to be $\eta$-subdifferentiable at a point $x \in H$ if there exists a point $f^{*} \in H$ such that

$$
\left\langle f^{*}, \eta(y, x)\right\rangle \leq \phi(y)-\phi(x) \quad \forall y \in H
$$

where $f^{*}$ is called an $\eta$-subgradient of $\phi$ at $x$. The set of all $\eta$-subgradients of $\phi$ at $x$ is denoted by $\partial \phi(x)$. The mapping $\partial \phi: H \rightarrow 2^{H}$ is defined by

$$
\partial \phi(x)=\left\{f^{*} \in H: \phi(y)-\phi(x) \geq\left\langle f^{*}, \eta(y, x)\right\rangle, \forall y \in H\right\} .
$$

DEFINITION 1.2 [1]. Let $\phi: H \rightarrow \mathbb{R} \cup\{+\infty\}$ be a proper functional and $\eta: H \times H \rightarrow H$ be a mapping. For any given $x \in H$ and any $\rho>0$, if there exists a unique point $u \in H$ such that

$$
\langle u-x, \eta(y, u)\rangle \geq \rho \phi(x)-\rho \phi(y) \quad \forall y \in H,
$$

then the mapping $x \rightarrow u$, denoted by $J_{\rho}^{\partial \phi}(x)$, is said to be an $\eta$-proximal mapping of $\phi$. By (1.5) and the definition of $J_{\rho}^{\partial \phi}(x)$, it is deduced that $x-u \in \rho \partial \phi(u)$. It follows that $J_{\rho}^{\partial \phi}(x)=(I+\rho \partial \phi)^{-1}(x)$, where $I$ is the identity mapping on $H$.

Definition 1.3 [1]. A functional $f: H \times H \rightarrow \mathbb{R} \cup\{+\infty\}$ is said to be 0-diagonally quasiconcave (0-DQCV) in $x$ if for any finite set $\left\{x_{1}, x_{2}, \ldots, x_{n}\right\} \subset H$ and for any $y=$ $\sum_{i=1}^{n} \lambda_{i} x_{i}$ with $\lambda_{i} \geq 0$ and $\sum_{i=1}^{n} \lambda_{i}=1$,

$$
\min _{1 \leq i \leq n} f\left(x_{i}, y\right) \leq 0 .
$$

Definition 1.4. A mapping $g: H \rightarrow H$ is said to be

(i) strongly monotone if there exists a constant $r>0$ such that

$$
\langle g(x)-g(y), x-y\rangle \geq r\|x-y\|^{2} \quad \forall x, y \in H,
$$

(ii) Lipschitz continuous if there exists a constant $r>0$ such that

$$
\|g(x)-g(y)\| \leq r\|x-y\| \quad \forall x, y \in H .
$$


DEFINITION 1.5. A mapping $\eta: H \times H \rightarrow H$ is said to be

(i) $\delta$-strongly monotone if there exists a constant $\delta>0$ such that

$$
\langle\eta(x, y), x-y\rangle \geq \delta\|x-y\|^{2} \quad \forall x, y \in H,
$$

(ii) $\tau$-Lipschitz continuous if there exists a contant $\tau>0$ such that

$$
\|\eta(x, y)\| \leq \tau\|x-y\| \quad \forall x, y \in H .
$$

DEFINITION 1.6 [2]. Let $T$ be a mapping from $H$ into $H, x_{0} \in H$, and let $x_{n+1}=$ $f\left(T, x_{n}\right)$ define an iterative procedure which yields a sequence of points $\left\{x_{n}\right\}_{n=0}^{\infty} \subseteq H$. Let $F(T)=\{x \in H: x=T x\} \neq \varnothing$. Suppose that $\left\{x_{n}\right\}_{n=0}^{\infty}$ converges to $q \in F(T)$. Let $\left\{y_{n}\right\}_{n=0}^{\infty} \subseteq H$ and let $\varepsilon_{n}=\left\|y_{n+1}-f\left(T, y_{n}\right)\right\|$ for each $n \geq 0$. If $\lim _{n \rightarrow \infty} \varepsilon_{n}=0$ implies that $\lim _{n \rightarrow \infty} y_{n}=q$, then the iteration procedure defined by $x_{n+1}=f\left(T, x_{n}\right)$ is said to be $T$-stable or stable with respect to $T$.

The following lemmas play an important role in proving our main results.

LEMMA 1.7 [1]. Let $\eta: H \times H \rightarrow H$ be $\delta$-strongly monotone and $\tau$-Lipschitz continuous such that $\eta(x, y)+\eta(y, x)=0$ for all $x, y \in H$ and for any given $x \in H$, the functional $h(y, u)=\langle x-u, \eta(y, u)\rangle$ is $0-D Q C V$ in $y$. Let $\phi: H \rightarrow \mathbb{R}$ be a lower semicontinuous $\eta$-subdifferentiable proper function and let $\rho>0$ be an arbitrary constant. Then the $\eta$-proximal mapping $J_{\rho}^{\partial \phi}$ of $\phi$ is $(\tau / \delta)$-Lipschitz continuous.

LEMMA 1.8 [5]. Let $\left\{\alpha_{n}\right\}_{n=0}^{\infty},\left\{\beta_{n}\right\}_{n=0}^{\infty}$, and $\left\{\gamma_{n}\right\}_{n=0}^{\infty}$ be three nonnegative sequences satisfying the following inequality:

$$
\alpha_{n+1} \leq\left(1-\omega_{n}\right) \alpha_{n}+\beta_{n} \omega_{n}+\gamma_{n} \quad \forall n \geq 0,
$$

where $\left\{\omega_{n}\right\}_{n=0}^{\infty} \subseteq[0,1], \sum_{n=0}^{\infty} \omega_{n}=\infty, \sum_{n=0}^{\infty} \gamma_{n}<\infty$, and $\lim _{n \rightarrow \infty} \beta_{n}=0$. Then $\lim _{n \rightarrow \infty} \alpha_{n}$ $=0$.

LEMMA 1.9. Let $\rho>0$ be a constant and $J_{\rho}^{\partial \phi(\cdot, x)}=(I+\partial \phi(\cdot, x))^{-1}$ for all $x \in H$. Then the following conditions are equivalent to each other:

(i) problem (1.1) has a solution $u \in H$;

(ii) there exists $u \in H$ satisfying the following relation:

$$
g(u)=m(u)+J_{\rho}^{\partial \phi(\cdot, u)}((g-m)(u)-\rho(A(u)-(B(u)-C(u)))) ;
$$

(iii) the mapping $T: H \rightarrow H$ defined by

$$
T x=x-(g-m)(x)+J_{\rho}^{\partial \phi(\cdot, x)}(f(x)) \quad \forall x \in H,
$$

has a fixed point $u \in H$, where

$$
f(x)=(g-m)(x)-\rho(A(x)-(B(x)-C(x))) \quad \forall x \in H .
$$

Proof. It is clear that (ii) and (iii) are equivalent. Note that (ii) holds if and only if there exists $u \in H$ satisfying

$$
-A(u)+B(u)-C(u) \in \partial \phi((g-m)(u), u),
$$


which is equivalent to finding $u \in H$ such that

$$
\langle A(u)-(B(u)-C(u)), \eta(y,(g-m)(u))\rangle \geq \phi((g-m)(u), u)-\phi(y, u) \quad \forall y \in H .
$$

That is, (i) and (ii) are equivalent. This completes the proof.

REMARK 1.10. Theorem 3.1 in [1] is a special case of Lemma 1.9.

Based on Lemma 1.9 we suggest the following three-step perturbed iterative algorithms.

Algorithm 1.11. Let $A, B, C, g, m: H \rightarrow H, \eta: H \times H \rightarrow H$ be mappings and let $\phi_{n}: H \times H \rightarrow \mathbb{R}$ be functional for each $n \geq 0$. Suppose that for each given $x \in H$ and $n \geq 0$, the $\eta$-proximal mapping of $\phi_{n}(\cdot, x)$ exists. For any given $u_{0} \in H$, the three-step perturbed iterative sequence $\left\{u_{n}\right\}_{n=0}^{\infty} \subseteq H$ is defined by

$$
\begin{aligned}
w_{n} & =\left(1-c_{n}\right) u_{n}+c_{n}\left[u_{n}-(g-m)\left(u_{n}\right)+J_{\rho}^{\partial \phi_{n}\left(\cdot, u_{n}\right)}\left(f\left(u_{n}\right)\right)\right]+p_{n}, \\
v_{n} & =\left(1-b_{n}\right) u_{n}+b_{n}\left[w_{n}-(g-m)\left(w_{n}\right)+J_{\rho}^{\partial \phi_{n}\left(\cdot, w_{n}\right)}\left(f\left(w_{n}\right)\right)\right]+q_{n}, \\
u_{n+1} & =\left(1-a_{n}\right) u_{n}+a_{n}\left[v_{n}-(g-m)\left(v_{n}\right)+J_{\rho}^{\partial \phi_{n}\left(\cdot, v_{n}\right)}\left(f\left(v_{n}\right)\right)\right]+r_{n}, \quad n \geq 0,
\end{aligned}
$$

where $f$ is defined by (1.15), $\left\{p_{n}\right\}_{n=0}^{\infty},\left\{q_{n}\right\}_{n=0}^{\infty},\left\{r_{n}\right\}_{n=0}^{\infty}$ are any sequences in $H$ and $\left\{a_{n}\right\}_{n=0}^{\infty},\left\{b_{n}\right\}_{n=0}^{\infty},\left\{c_{n}\right\}_{n=0}^{\infty}$ are arbitrary sequences in $[0,1]$ satisfying

$$
\sum_{n=0}^{\infty} a_{n}=\infty .
$$

Algorithm 1.12. Let $A, B, C, g, m: H \rightarrow H, \eta: H \times H \rightarrow H$ be mappings and let $\phi: H \times H \rightarrow \mathbb{R}$ be a functional. Suppose that for each given $x \in H$, the $\eta$-proximal mapping of $\phi(\cdot, x)$ exists. For any given $u_{0} \in H$, the three-step iterative sequence with errors $\left\{u_{n}\right\}_{n=0}^{\infty} \subseteq H$ is defined by

$$
\begin{aligned}
w_{n} & =\left(1-c_{n}\right) u_{n}+c_{n} T\left(u_{n}\right)+p_{n}, \\
v_{n} & =\left(1-b_{n}\right) u_{n}+b_{n} T\left(w_{n}\right)+q_{n}, \\
u_{n+1} & =\left(1-a_{n}\right) u_{n}+a_{n} T\left(v_{n}\right)+r_{n}, \quad n \geq 0,
\end{aligned}
$$

where $T$ is defined by (1.14), $\left\{p_{n}\right\}_{n=0}^{\infty},\left\{q_{n}\right\}_{n=0}^{\infty},\left\{r_{n}\right\}_{n=0}^{\infty}$ are any sequences in $H$, and $\left\{a_{n}\right\}_{n=0}^{\infty},\left\{b_{n}\right\}_{n=0}^{\infty},\left\{c_{n}\right\}_{n=0}^{\infty}$ are arbitrary sequences in [0,1] satisfying (1.19).

REMARK 1.13. Algorithms 3.3 and 3.4 in [1] are special cases of Algorithm 1.11.

\section{Convergence and stability}

THEOREM 2.1. Let $A, B, C, g$, and $m: H \rightarrow H$ be Lipschitz continuous mappings with constants $\beta, \gamma, \sigma, \nu$, and $\zeta$, respectively, and let $A$ and $(g-m)$ be strongly monotone with constants $\alpha$ and $\xi$, respectively. Let $\eta: H \times H \rightarrow H$ be strongly monotone and Lipschitz 
with constants $\delta$ and $\tau$, respectively, satisfying $\eta(x, y)+\eta(y, x)=0$ for any $x, y \in$ $H$, and for each given $x \in H$, the functional $h(y, u)=\langle x-u, \eta(y, u)\rangle$ is $0-D Q C V$ in $y$. Suppose that $\phi: H \times H \rightarrow \mathbb{R}$ satisfies that for each fixed $y \in H, \phi(\cdot, u)$ is a lower semicontinuous $\eta$-subdifferentiable proper functional with $(g-m)(H) \cap \operatorname{dom} \partial \phi(\cdot, y) \neq$ $\varnothing$. Assume that there exists a constant $\mu>0$ such that

$$
\left\|J_{\rho}^{\partial \phi(\cdot, x)}(z)-J_{\rho}^{\partial \phi(\cdot, y)}(z)\right\| \leq \mu\|x-y\| \quad \forall x, y, z \in H .
$$

Let $k=\mu+(1+\tau / \delta) \sqrt{1-2 \xi+(\nu+\zeta)^{2}}$. If there exists a constant $\rho>0$ satisfying

$$
\rho<\frac{(1-k) \delta}{\tau(\gamma+\sigma)}
$$

and one of the following conditions:

$$
\begin{aligned}
& \beta>\gamma+\sigma, \\
& \tau \alpha>\delta(1-k)(\gamma+\sigma)+\sqrt{\left[\beta^{2}-(\gamma+\sigma)^{2}\right]\left[\tau^{2}-(1-k)^{2} \delta^{2}\right]}, \\
& \left|\rho-\frac{\delta(k-1)(\gamma+\sigma)+\tau \alpha}{\tau\left[\beta^{2}-(\gamma+\sigma)^{2}\right]}\right| \\
& \quad<\frac{\sqrt{[\tau \alpha-\delta(1-k)(\gamma+\sigma)]^{2}-\left[\beta^{2}-(\gamma+\sigma)^{2}\right]\left[\tau^{2}-\delta^{2}(1-k)^{2}\right]}}{\tau\left[\beta^{2}-(\gamma+\sigma)^{2}\right]} ; \\
& \beta=\gamma+\sigma, \quad \tau \alpha>\delta(1-k) \beta, \quad \rho>\frac{\delta^{2}(1-k)^{2}-\tau^{2}}{2 \tau[\tau-(1-k) \delta(\gamma+\sigma)]} ; \\
& \beta<\gamma+\sigma, \quad \frac{(1-k) \delta(\gamma+\sigma)-\tau \alpha}{\tau\left[(\gamma+\sigma)^{2}-\beta^{2}\right]} \mid \\
& \quad>\frac{\sqrt{[\tau \alpha-\delta(1-k)(\gamma+\sigma)]^{2}+\left[(\gamma+\sigma)^{2}-\beta^{2}\right]\left[\tau^{2}-(1-k)^{2} \delta^{2}\right]}}{\tau\left[(\gamma+\sigma)^{2}-\beta^{2}\right]},
\end{aligned}
$$

then problem (1.1) has a unique solution $u \in H$.

Proof. It follows from the strong monotonicity of $A$ and $(g-m)$ and Lipschitz continuity of $A, g$, and $m$ that

$$
\begin{aligned}
\| x- & y-((g-m)(x)-(g-m)(y)) \|^{2} \\
& =\|x-y\|^{2}-2\langle x-y,(g-m)(x)-(g-m)(y)\rangle+\|(g-m)(x)-(g-m)(y)\|^{2} \\
& \leq\left[1-2 \xi+(v+\zeta)^{2}\right]\|x-y\|^{2}, \\
\| x- & y-\rho(A(x)-B(y)) \|^{2} \\
& =\|x-y\|^{2}-2 \rho\langle x-y, A(x)-A(y)\rangle+\|A(x)-A(y)\|^{2} \\
& \leq\left(1-2 \rho \alpha+\rho^{2} \beta^{2}\right)\|x-y\|^{2},
\end{aligned}
$$


for any $x, y \in H$. In light of (1.14), (2.6), Lemma 1.7, and the Lipschitz continuity of $B$ and $C$, we infer that for any $x, y \in H$,

$$
\begin{aligned}
\|T x-T y\| \leq & \|x-y-((g-m)(x)-(g-m)(y))\| \\
& +\left\|J_{\rho}^{\partial \phi(\cdot, x)}(f(x))-J_{\rho}^{\partial \phi(\cdot, x)}(f(y))\right\| \\
& +\left\|J_{\rho}^{\partial \phi(\cdot, x)}(f(y))-J_{\rho}^{\partial \phi(\cdot, y)}(f(y))\right\| \\
\leq & \left(1+\frac{\tau}{\delta}\right)\|x-y-((g-m)(x)-(g-m)(y))\| \\
& +\frac{\tau}{\delta}\|x-y-\rho(A(x)-A(y))\|+\frac{\rho \tau}{\delta}\|B(x)-B(y)\| \\
& +\frac{\rho \tau}{\delta}\|C(x)-C(y)\|+\mu\|x-y\| \\
\leq & \theta\|x-y\|,
\end{aligned}
$$

where

$$
\theta=k+\frac{\tau}{\delta} \sqrt{1-2 \rho \alpha+\rho^{2} \beta^{2}}+\frac{\rho \tau(\gamma+\sigma)}{\delta} .
$$

Thus (2.2) and one of (2.3)-(2.5) ensure that $\theta<1$. Hence $T$ is a contraction mapping and it has a unique fixed point $u \in H$. Lemma 1.9 means that $u$ is a unique solution of problem (1.1). This completes the proof.

REMARK 2.2. Theorem 2.1 extends and improves [1, Theorem 3.6].

Next we show convergence of the three-step perturbed iterative sequence and threestep iterative sequence with errors generated by Algorithms 1.11 and 1.12, respectively.

THEOREM 2.3. Let $A, B, C, g, m, \eta$, and $\phi$ be as in Theorem 2.1 satisfying (2.1), (2.2), and one of the conditions (2.3)-(2.5). Let $\phi_{n}: H \times H \rightarrow \mathbb{R}$ be such that for any fixed $y \in H$ and $n \geq 0, \phi_{n}(\cdot, y): H \rightarrow \mathbb{R}$ is lower semicontinuous $\eta$-subdifferentiable on $H$. Assume that

$$
\begin{gathered}
\left\|J_{\rho}^{\partial \phi_{n}(\cdot, x)}(z)-J_{\rho}^{\partial \phi_{n}(\cdot, y)}(z)\right\| \leq \mu\|x-y\| \quad \forall x, y, z \in H, n \geq 0, \\
\lim _{n \rightarrow \infty}\left\|J_{\rho}^{\partial \phi_{n}(\cdot, x)}(y)-J_{\rho}^{\partial \phi(\cdot, x)}(y)\right\|=0 \quad \forall x, y \in H, \\
\lim _{n \rightarrow \infty} b_{n}\left\|p_{n}\right\|=\lim _{n \rightarrow \infty}\left\|q_{n}\right\|=0 .
\end{gathered}
$$

If one of the conditions

(C1) $\sum_{n=0}^{\infty}\left\|r_{n}\right\|<\infty$,

(C2) there exists a nonnegative sequence $\left\{d_{n}\right\}_{n=0}^{\infty}$ satisfying $\lim _{n \rightarrow \infty} d_{n}=0$ and $\left\|r_{n}\right\|=$ $a_{n} d_{n}$ for all $n \geq 0$,

is fulfilled, then the three-step perturbed iterative sequence $\left\{u_{n}\right\}_{n=0}^{\infty}$ generated by Algorithm 1.11 converges strongly to the unique solution of problem (1.1). 
Proof. It follows from Theorem 2.1 that problem (1.1) has a unique solution $u \in H$. That is, $u=T u$, where $T$ is defined by (1.14). Notice that

$$
u=\left(1-a_{n}\right) u+a_{n} T(u)=\left(1-b_{n}\right) u+b_{n} T(u)=\left(1-c_{n}\right) u+c_{n} T(u) \quad \forall n \geq 0 .
$$

Using the same argument as in the proof of Theorem 2.1, from (2.9), (2.12), and Lemma 1.7 we obtain that for any $n \geq 0$,

$$
\begin{aligned}
\left\|w_{n}-u\right\|= & \|\left(1-c_{n}\right) u_{n}+c_{n}\left[u_{n}-(g-m)\left(u_{n}\right)+J_{\rho}^{\partial \phi_{n}\left(\cdot, u_{n}\right)}\left(f\left(u_{n}\right)\right)\right] \\
& +p_{n}-\left(1-c_{n}\right) u-c_{n}\left[u-(g-m)(u)+J_{\rho}^{\partial \phi(\cdot, u)}(f(u))\right] \| \\
\leq & \left(1-c_{n}\right)\left\|u_{n}-u\right\|+c_{n}\left\|u_{n}-u-\left((g-m)\left(u_{n}\right)-(g-m)(u)\right)\right\| \\
& +c_{n}\left\|J_{\rho}^{\partial \phi_{n}\left(\cdot, u_{n}\right)}\left(f\left(u_{n}\right)\right)-J_{\rho}^{\partial \phi_{n}\left(\cdot, u_{n}\right)}(f(u))\right\| \\
& +c_{n}\left\|J_{\rho}^{\partial \phi_{n}\left(\cdot, u_{n}\right)}(f(u))-J_{\rho}^{\partial \phi_{n}(\cdot, u)}(f(u))\right\| \\
& +c_{n}\left\|J_{\rho}^{\partial \phi_{n}(\cdot, u)}(f(u))-J_{\rho}^{\partial \phi(\cdot, u)}(f(u))\right\|+\left\|p_{n}\right\| \\
\leq & \left(1-c_{n}\right)\left\|u_{n}-u\right\|+c_{n}\left\|u_{n}-u-\left((g-m)\left(u_{n}\right)-(g-m)(u)\right)\right\| \\
& +c_{n} \frac{T}{\delta}\left\|f\left(u_{n}\right)-f(u)\right\|+c_{n} \mu\left\|u_{n}-u\right\|+c_{n} \varepsilon_{n}+\left\|p_{n}\right\| \\
\leq & \left(1-c_{n}\right)\left\|u_{n}-u\right\|+c_{n} \theta\left\|u_{n}-u\right\|+c_{n} \varepsilon_{n}+\left\|p_{n}\right\|,
\end{aligned}
$$

where $\theta$ satisfies (2.8) and $\varepsilon_{n}=\left\|J_{\rho}^{\partial \phi_{n}(\cdot, u)}(f(u))-J_{\rho}^{\partial \phi(\cdot, u)}(f(u))\right\|$. It follows from (2.2) and one of (2.3)-(2.5) that $\theta<1$. In view of (2.13) we know that

$$
\left\|w_{n}-u\right\| \leq\left\|u_{n}-u\right\|+c_{n} \varepsilon_{n}+\left\|p_{n}\right\| \quad \forall n \geq 0 .
$$

Similarly we infer that

$$
\begin{gathered}
\left\|v_{n}-u\right\| \leq\left\|u_{n}-u\right\|+2 b_{n} \varepsilon_{n}+b_{n}\left\|p_{n}\right\|+\left\|q_{n}\right\| \quad \forall n \geq 0, \\
\left\|u_{n+1}-u\right\| \leq\left(1-a_{n}(1-\theta)\right)\left\|u_{n}-u\right\|+3 a_{n} \varepsilon_{n}+a_{n} b_{n}\left\|p_{n}\right\|+a_{n}\left\|q_{n}\right\|+\left\|r_{n}\right\| \quad \forall n \geq 0 .
\end{gathered}
$$

Suppose that (C1) holds. Let

$$
\begin{gathered}
\alpha_{n}=\left\|u_{n}-u\right\|, \quad \omega_{n}=(1-\theta) a_{n}, \\
\beta_{n}=(1-\theta)^{-1}\left(3 \varepsilon_{n}+b_{n}\left\|p_{n}\right\|+\left\|q_{n}\right\|\right), \quad \gamma_{n}=\left\|r_{n}\right\| \quad \forall n \geq 0 .
\end{gathered}
$$

It follows from Lemma 1.8, (1.19), (2.10), (2.11), (C1), and (2.16) that $\left\{u_{n}\right\}_{n=0}^{\infty}$ converges strongly to $u$.

Suppose that (C2) holds. Set

$$
\begin{gathered}
\alpha_{n}=\left\|u_{n}-u\right\|, \quad \omega_{n}=(1-\theta) a_{n}, \\
\beta_{n}=(1-\theta)^{-1}\left(3 \varepsilon_{n}+b_{n}\left\|p_{n}\right\|+\left\|q_{n}\right\|+d_{n}\right), \quad \gamma_{n}=0, \quad \forall n \geq 0 .
\end{gathered}
$$


Thus Lemma 1.8, (1.19), (2.10), (2.11), (C2), and (2.16) ensure that $\left\{u_{n}\right\}_{n=0}^{\infty}$ converges strongly to $u$. This completes the proof.

In case $\phi_{n}=\phi$ for all $n \geq 0$, then Theorem 2.3 reduces to the following.

THEOREM 2.4. Let $A, B, C, g, m, \eta$, and $\phi$ be as in Theorem 2.1 satisfying (2.1), (2.2), (2.11), and one of the conditions (2.3)-(2.5). If either (C1) or (C2) holds, then the three-step iterative sequence with errors $\left\{u_{n}\right\}_{n=0}^{\infty}$ generated by Algorithm 1.12 converges strongly to the unique solution of problem (1.1).

Now we study stability of the three-step iterative sequence with errors generated by Algorithm 1.12.

THEOREM 2.5. Let $g, m, A, B, C$, $\eta$, and $\phi$ be as in Theorem 2.4 satisfying (2.1), (2.2), (2.11), and one of the conditions (2.3)-(2.5). Let $\left\{y_{n}\right\}_{n=0}^{\infty}$ be any sequence in $H$ and define $\left\{\varepsilon_{n}\right\}_{n=0}^{\infty} \subseteq[0, \infty)$ by

$$
\begin{aligned}
\varepsilon_{n} & =\left\|y_{n+1}-\left(1-a_{n}\right) y_{n}-a_{n} T x_{n}-r_{n}\right\|, \\
x_{n} & =\left(1-b_{n}\right) y_{n}+b_{n} T z_{n}+q_{n}, \\
z_{n} & =\left(1-c_{n}\right) y_{n}+c_{n} T y_{n}+p_{n} \quad \forall n \geq 0,
\end{aligned}
$$

where $T$ satisfies (1.14). If there exists a constant $d>0$ satisfying

$$
a_{n} \geq d \quad \forall n \geq 0,
$$

and if

$$
\lim _{n \rightarrow \infty}\left\|r_{n}\right\|=0
$$

then the three-step iterative sequence with errors $\left\{u_{n}\right\}_{n=0}^{\infty}$ generated by Algorithm 1.12 converges strongly to the unique solution $u \in H$ of problem (1.1). Moreover, $\lim _{n \rightarrow \infty} y_{n}=$ $u$ if and only if $\lim _{n \rightarrow \infty} \varepsilon_{n}=0$.

Proof. Set $d_{n}=\left\|r_{n}\right\| / a_{n}$ for all $n \geq 0$. Then (2.21) implies that (C2) holds. Obviously, (2.20) yields that (1.19) holds. It follows from Theorem 2.4 that the three-step iterative sequence with errors $\left\{u_{n}\right\}_{n=0}^{\infty}$ generated by Algorithm 1.12 converges strongly to the unique solution $u$ of problem (1.1).

Put $y_{n+1}-\left(1-a_{n}\right) y_{n}-a_{n} T x_{n}-r_{n}=h_{n}$ for all $n \geq 0$. Then $y_{n+1}=\left(1-a_{n}\right) y_{n}+$ $a_{n} T x_{n}+r_{n}+h_{n}$ and $\varepsilon_{n}=\left\|h_{n}\right\|$ for all $n \geq 0$. As in the proof of Theorem 2.3, we deduce that

$$
\begin{aligned}
& \left\|\left(1-a_{n}\right) y_{n}+a_{n} T x_{n}+r_{n}-u\right\| \leq(1-(1-\theta) d)\left\|y_{n}-u\right\|+b_{n}\left\|p_{n}\right\|+\left\|q_{n}\right\|+\left\|r_{n}\right\| \\
& \begin{aligned}
\left\|y_{n+1}-u\right\| & \leq\left(1-(1-\theta) a_{n}\right)\left\|y_{n}-u\right\|+a_{n} b_{n}\left\|p_{n}\right\|+a_{n}\left\|q_{n}\right\|+\left\|r_{n}\right\|+\left\|h_{n}\right\| \\
& \leq(1-(1-\theta) d)\left\|y_{n}-u\right\|+b_{n}\left\|p_{n}\right\|+\left\|q_{n}\right\|+\left\|r_{n}\right\|+\varepsilon_{n}
\end{aligned}
\end{aligned}
$$

for all $n \geq 0$. 
Suppose that $\lim _{n \rightarrow \infty} \varepsilon_{n}=0$. Let

$$
\begin{gathered}
\alpha_{n}=\left\|y_{n}-u\right\|, \quad \omega_{n}=(1-\theta) d, \\
\beta_{n}=\omega_{n}^{-1}\left(b_{n}\left\|p_{n}\right\|+\left\|q_{n}\right\|+\left\|r_{n}\right\|+\varepsilon_{n}\right), \quad \gamma_{n}=0 \quad \forall n \geq 0 .
\end{gathered}
$$

Then Lemma 1.8, (2.11), (2.21), and (2.23) ensure that $\lim _{n \rightarrow \infty} y_{n}=u$.

Suppose that $\lim _{n \rightarrow \infty} y_{n}=u$. It follows from (2.11), (2.21), and (2.22) that

$$
\begin{aligned}
\varepsilon_{n} & \leq\left\|y_{n+1}-u\right\|+\left\|\left(1-a_{n}\right) y_{n}+a_{n} T x_{n}+r_{n}-u\right\| \\
& \leq\left\|y_{n+1}-u\right\|+(1-(1-\theta) d)\left\|y_{n}-u\right\|+b_{n}\left\|p_{n}\right\|+\left\|q_{n}\right\|+\left\|r_{n}\right\| \\
& \longrightarrow 0
\end{aligned}
$$

as $n \rightarrow \infty$. That is, $\lim _{n \rightarrow \infty} \varepsilon_{n}=0$. This completes the proof.

ACKNOWLEDGMENT. This work was supported by the Korea Research Foundation Grant KRF-2003-002-C00018.

\section{REFERENCES}

[1] X. P. Ding and C. L. Luo, Perturbed proximal point algorithms for general quasi-variationallike inclusions, J. Comput. Appl. Math. 113 (2000), no. 1-2, 153-165.

[2] A. M. Harder, Fixed point theory and stability results for fixed point iteration procedures, Ph.D. thesis, University of Missouri-Rolla, Missouri, 1987.

[3] A. Hassouni and A. Moudafi, A perturbed algorithm for variational inclusions, J. Math. Anal. Appl. 185 (1994), no. 3, 706-712.

[4] N.-J. Huang, Generalized nonlinear variational inclusions with noncompact valued mappings, Appl. Math. Lett. 9 (1996), no. 3, 25-29.

[5] L. S. Liu, Ishikawa and Mann iterative process with errors for nonlinear strongly accretive mappings in Banach spaces, J. Math. Anal. Appl. 194 (1995), no. 1, 114-125.

[6] Z. Liu, L. Debnath, S. M. Kang, and J. S. Ume, Sensitivity analysis for parametric completely generalized nonlinear implicit quasivariational inclusions, J. Math. Anal. Appl. 277 (2003), no. 1, 142-154.

[7] Z. Liu, S. M. Kang, and J. S. Ume, Completely generalized multivalued strongly quasivariational inequalities, Publ. Math. Debrecen 62 (2003), no. 1-2, 187-204.

[8] Z. Liu, J. S. Ume, and S. M. Kang, General strongly nonlinear quasivariational inequalities with relaxed Lipschitz and relaxed monotone mappings, J. Optim. Theory Appl. 114 (2002), no. 3, 639-656.

[9] _ Resolvent equations technique for general variational inclusions, Proc. Japan Acad. Ser. A Math. Sci. 78 (2002), no. 10, 188-193.

Zeqing Liu: Department of Mathematics, Liaoning Normal University, P. O. Box 200, Dalian, Liaoning 116029, China

E-mail address: zeqing $7 \mathrm{u}$ @d 1 .cn

Zhefu An: Department of Mathematics, Liaoning University, Shenyang, Liaoning 110036, China E-mail address: zhefuan@yahoo.com.cn

Shin Min Kang: Department of Mathematics and Research Institute of Natural Science, Gyeongsang National University, Chinju 660-701, Korea

E-mail address: smkang@nongae.gsnu.ac. kr

Jeong Sheok Ume: Department of Applied Mathematics, Changwon National University, Changwon 641-773, Korea

E-mail address: jsume@sarim. changwon.ac. kr 


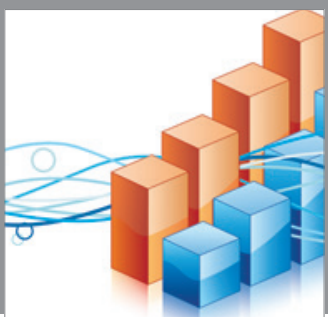

Advances in

Operations Research

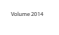



\section{The Scientific} World Journal


International Journal of

Mathematics and

Mathematical

Sciences
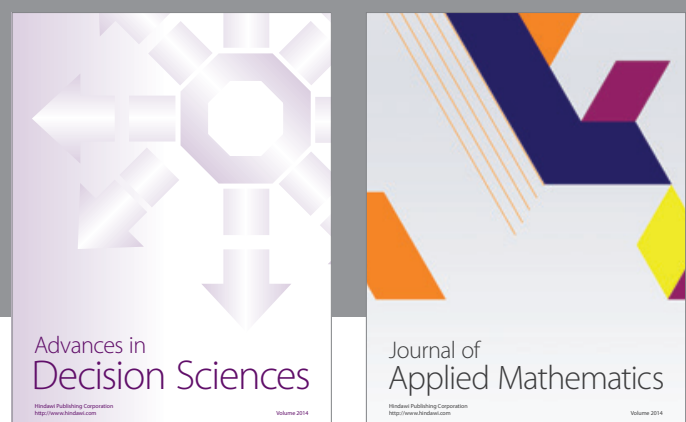

Journal of

Applied Mathematics
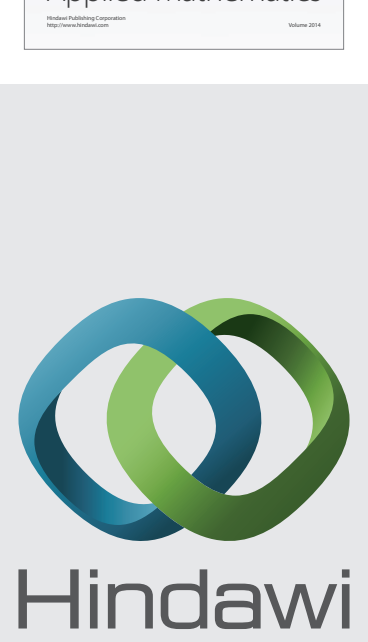

Submit your manuscripts at http://www.hindawi.com
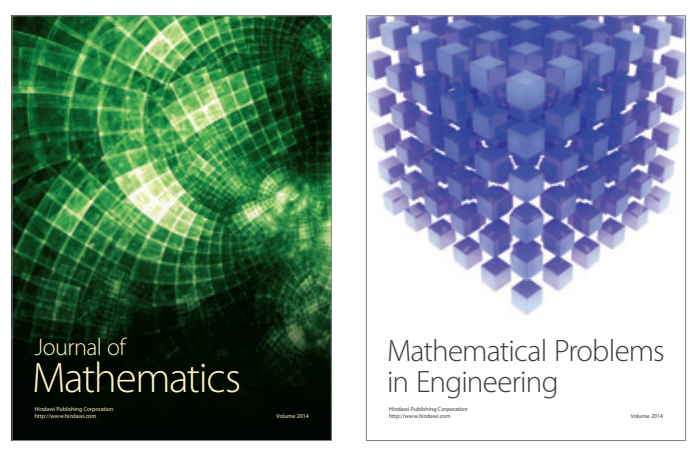

Mathematical Problems in Engineering
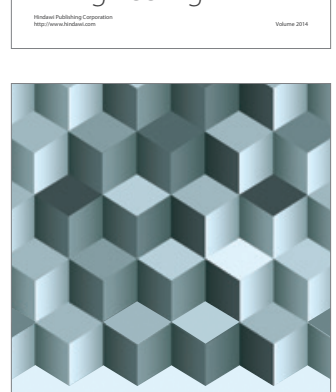

Journal of

Function Spaces
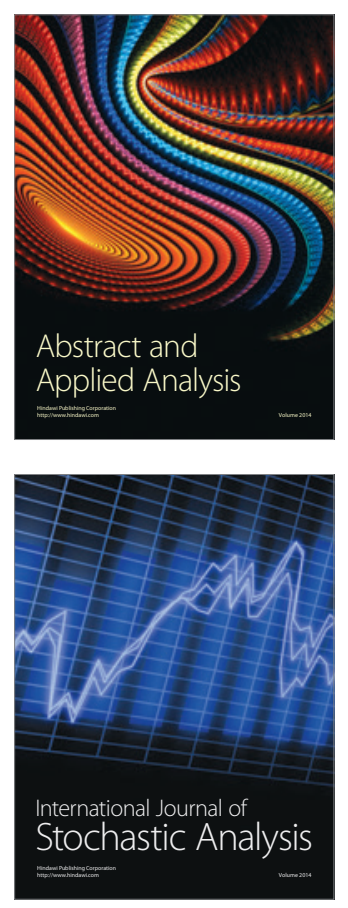

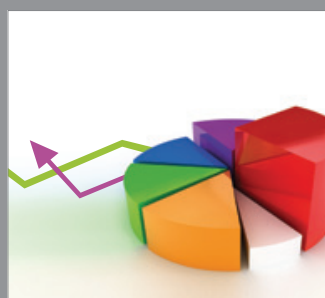

ournal of

Probability and Statistics

Promensencen
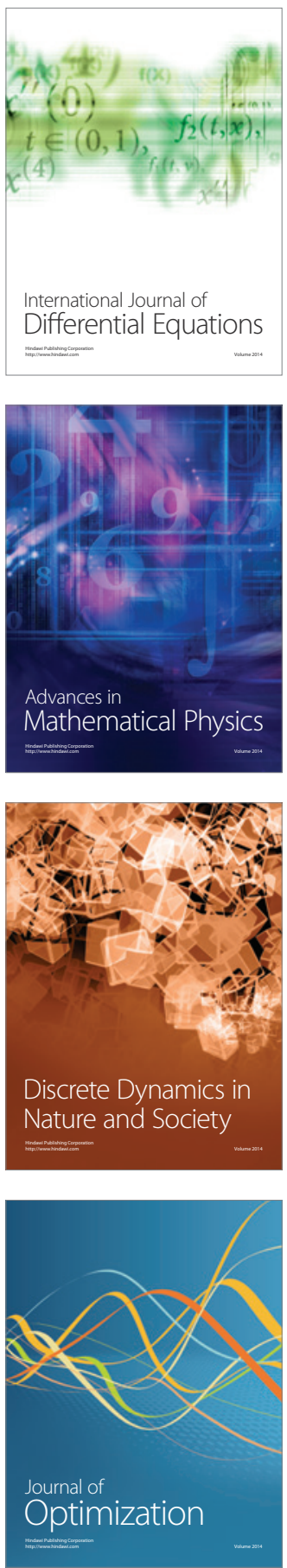